\title{
Splice Loss of Graded-Index Fibers: Accurate Semianalytical Descriptions Using Nelder-Mead Nonlinear Unconstrained Optimization with Three-Parameter Fundamental Modal Field
}

\author{
Raja Roy Choudhury, ${ }^{1}$ Arundhati Roy Choudhury, ${ }^{2}$ and Mrinal Kanti Ghose ${ }^{3}$ \\ ${ }^{1}$ Applied Electronics and Instrumentation Department, Sikkim Manipal Institute of Technology, Majitar, Sikkim 737136, India \\ ${ }^{2}$ Physics Department, Sikkim Manipal Institute of Technology, Majitar, Sikkim 737136, India \\ ${ }^{3}$ Computer Science Department, Sikkim Manipal Institute of Technology, Majitar, Sikkim 737136, India
}

Correspondence should be addressed to Raja Roy Choudhury; ra_ch2@yahoo.co.in

Received 5 March 2013; Accepted 4 June 2013

Academic Editor: Yong Zhao

Copyright (C) 2013 Raja Roy Choudhury et al. This is an open access article distributed under the Creative Commons Attribution License, which permits unrestricted use, distribution, and reproduction in any medium, provided the original work is properly cited.

A faster and accurate semianalytical formulation with a robust optimization solution for estimating the splice loss of graded-index fibers has been proposed. The semianalytical optimization of modal parameters has been carried out by Nelder-Mead method of nonlinear unconstrained minimization suitable for functions which are uncertain, noisy, or even discontinuous. Instead of normally used Gaussian function, as the trial field for the fundamental mode of graded-index optical fiber a novel sinc function with exponentially and $R^{-3 / 2}$ ( $R$ is the normalized radius of the optical fiber) decaying trailing edge has been used. Due to inclusion of three parameters in the optimization of fundamental modal solution and application of an efficient optimization technique with simple analytical expressions for various modal parameters, the results are found to be accurate and computationally easier to find than the standard numerical method solution.

\section{Introduction}

Single mode fiber is considered as the most important broadband transmission media for optical communication system. Achieving accurate values of modal field distribution in such fiber is very essential, as it can provide basic solutions for wave equation and many useful properties like splice loss, microbending loss, fiber coupling, and the prediction of intramodal dispersion [1]. However, the various expressions for the fundamental modal field that have been reported so far are not able to predict propagation constant and modal parameters exactly in all regions of single mode operation [2]. The Gaussian approximation shows poor accuracy for lower normalized frequency region although this region may involve single mode fiber operation [2]; however, it can perform satisfactorily only for higher normalized frequency region and give good result near the cut-off frequency of next higher mode [3]. Besides, it is also equally important that the approximation should describe the field in the cladding accurately, as it is useful in the study of evanescent coupling problem. To overcome these inefficiencies, an exponentially and $R^{-3 / 2}$ decaying trailing edge fundamental modal field solution in core-cladding interface region has been considered.

To achieve higher accuracy compared to Gaussian function, the Gaussian-Hankel [2], the generalized Gaussian [4], the extended Gaussian [5], and the Laguerre-Gauss/Bessel expansion approximation $[6,7]$ have been proposed so far. An approximate analytical description with no requirement for optimization has also been presented [8]. But such analytical expression may not work for all specifications of an optical fiber. In the proposed formulation, Nelder-Mead method of nonlinear unconstrained minimization and the process of minimization of core parameter $(U)$ for all specific requirements have been used to achieve an accurate and computationally appropriate result.

Unlike the existing reported fundamental modal solution with one or two parameters [2-8], an attempt has been 
made to propose a three-parameter fundamental modal field solution for graded-index fiber to introduce more flexibility to solve the fundamental modal solution more accurately, especially in core-cladding interface region wherein the solution has different form (exponentially and $R^{-3 / 2}$ decaying trailing edge). Ghatak et al. [9] had arrived at simple analytical expressions to describe different optical fiber characteristics by implementing variational technique. Again, the optimization process requires expressions for propagation constant $\beta$ and core parameter $U$. The analytical expressions for $\beta$ and $U$ used for the present study involve many fiber parameters, such as core radius $(a)$, refractive indices of core and cladding $\left(n_{\mathrm{co}}\right.$ and $\left.n_{\mathrm{cl}}\right)$, aspect ratio $\left(S_{0}\right)$, and wavelength $(\lambda=2 \pi / k)$, where $k$ is the free space wave number. Hence, any desired specification can be incorporated by varying these parameters. Now, the task of optimization can be carried out by using Nelder-Mead method of nonlinear unconstrained minimization, to meet a particular design.

For graded-index optical fiber at the splices, the power transmission coefficients with transverse and angular mismatch have been estimated by using the methods given by Meunier and Hosain [10] and Hosain et al. [11]. For arbitrarily graded-index fiber, the Gaussian approximation does not give accurate result at lower normalized frequency or in cases where the power law profile deviates from its simplest form [12]. Further, the numerical solution requires rigorous computations and specialized numerical techniques [13]. However, using the proposed three-parameter fundamental modal solution coupled with Nelder-Mead method of nonlinear unconstrained minimization, the algorithm becomes comparatively easier to be implemented on an ordinary personal computer, which provides computationally more efficient result $[14,15]$ than standard numerical method and yields excellent agreement with exact solutions. This is achieved due to the fact that requisite analytical formulae are deduced beforehand and then parameters of those analytical expressions are found by optimization using Nelder-Mead simplex method for nonlinear unconstrained minimization. Furthermore, Nelder-Mead simplex method for nonlinear unconstrained minimization is a direct search method $[16,17]$ which does not require any derivative information, so it can optimize nonstationary functions, as needed for the problems under study [18-20]. The proposed semianalytical model can also be used in the study of nonlinear fiber [21].

\section{Formulation of the Problem}

2.1. Theory. Splice loss can be evaluated analytically with the help of the following equations [22]:

$$
\begin{gathered}
\int\left|\psi_{1}\right|^{2} R d R=\frac{\log \left(\alpha R / R_{0}\right)-C i\left(2 \alpha R / R_{0}\right)}{2}, \\
\int\left|\psi_{2}\right|^{2} R d R=\frac{\sin ^{2} \alpha e^{2 \mu}}{R}\left(-R_{0} e^{-2 \mu R / R_{0}}+2 \mu R E i\left(1, \frac{2 \mu R}{R_{0}}\right)\right),
\end{gathered}
$$

$$
\begin{aligned}
& \int n_{f 2}^{2} R\left|\psi_{1}\right|^{2} d R \\
& =\frac{1}{\left(-4 \alpha+4 \alpha S_{0}\right)} \\
& \times\left[2 n_{2}^{2} \alpha S_{0} \log \left(\frac{\alpha R}{R_{0}}\right)-2 n_{2}^{2} \alpha S_{0} C i\left(\frac{2 \alpha R}{R_{0}}\right)\right. \\
& -2 n_{1}^{2} \alpha \log \left(\frac{\alpha R}{R_{0}}\right)+2 n_{1}^{2} \alpha C i\left(\frac{\alpha R}{R_{0}}\right) \\
& -n_{1}^{2} R_{0} \sin \left(\frac{2 \alpha R}{R_{0}}\right)+2 n_{1}^{2} R \alpha \\
& \left.+n_{2}^{2} R_{0} \sin \left(\frac{2 \alpha R}{R_{0}}\right)-2 n_{2}^{2} \alpha R\right] \text {, } \\
& \int n_{f 2}^{2} R\left|\psi_{2}\right|^{2} d R \\
& =\frac{e^{2 \mu} \sin ^{2} \alpha}{R\left(S_{0}-1\right)} \\
& \times\left[-n_{2}^{2} R_{0} S_{0} e^{-\left(2 \mu R / R_{0}\right)}+2 n_{2}^{2} \mu R S_{0} E i\left(1, \frac{2 \mu R}{R_{0}}\right)\right. \\
& +n_{1}^{2} R_{0} e^{-\left(2 \mu R / R_{0}\right)}-2 n_{1}^{2} \mu R E i\left(1, \frac{2 \mu R}{R_{0}}\right) \\
& \left.-n_{1}^{2} R_{0} R E i\left(1, \frac{2 \mu R}{R_{0}}\right)+n_{2}^{2} R_{0} R E i\left(1, \frac{2 \mu R}{R_{0}}\right)\right] \text {, } \\
& \int\left|\frac{d \psi_{1}}{d R}\right|^{2} R d R=\left[\frac{1}{2 R_{0}^{2}} \log \left(\frac{\alpha R}{R_{0}}\right)-\frac{1}{2 R_{0}^{2}} \operatorname{Ci}\left(\frac{2 \alpha R}{R_{0}}\right)\right] \alpha^{2} \\
& +\frac{\alpha}{2 R R_{0}} \sin \left(\frac{2 \alpha R}{R_{0}}\right) \\
& +\frac{1}{4 R^{2}} \cos \left(\frac{2 \alpha R}{R_{0}}\right)-\frac{1}{4 R^{2}} \\
& \int\left|\frac{d \psi_{2}}{d R}\right|^{2} R d R \\
& =-\frac{\sin ^{2} \alpha e^{2 \mu}}{4 R_{0}^{2} R^{3} e^{2 \mu R / R_{0}}}\left(3 R_{0}^{3}+3 R_{0}^{2} \mu R-2 R_{0} \mu^{2} R^{2}\right. \\
& \left.+4 E i\left(1, \frac{2 \mu R}{R_{0}}\right) \mu^{3} R^{3} e^{2 \mu R / R_{0}}\right), \\
& \int\left|\psi_{1}\right|^{2} R^{3} d R \\
& =\frac{1}{8 \alpha^{2}}\left[-2 \alpha R R_{0} \sin \left(\frac{2 \alpha R}{R_{0}}\right)\right. \\
& \left.+2 \alpha^{2} R^{2}+R_{0}^{2}-R_{0}^{2} \cos \left(\frac{2 \alpha R}{R_{0}}\right)\right],
\end{aligned}
$$




$$
\int\left|\psi_{2}\right|^{2} R^{3} d R=-\frac{\sin \alpha^{2} e^{2 \mu} R_{0}^{2}}{2 \mu e^{2 \mu R / R_{0}}},
$$

$$
\begin{aligned}
& \int\left|\psi_{1}\right|^{2} R^{q} d R \\
& =\frac{\alpha^{2}}{R_{0}^{2}(q+1)} R^{q+1} \\
& \quad \times \text { Hypergeom }\left(\left[1, \frac{q}{2}+\frac{1}{2}\right],\left[2, \frac{3}{2}, \frac{3}{2}+\frac{q}{2}\right],-\frac{\alpha^{2} R^{2}}{R_{0}^{2}}\right),
\end{aligned}
$$

$$
\begin{aligned}
& \int\left|\psi_{2}\right|^{2} R^{q} d R \\
&=2^{(2-q)}\left(\frac{\mu}{R_{0}}\right)^{-q} \frac{\mu^{2}}{R_{0}} e^{2 \mu} \\
& \times \sin \alpha^{2}\left[\frac{1}{(q-2)(q-1) q(q+1)}\left(\frac{\mu R}{R_{0}}\right)^{-q / 2}\right. \\
& \times e^{-\mu R / R_{0}} R^{q} 2^{q / 2}\left(\frac{\mu}{R_{0}}\right)^{q} \\
& \times W M\left(\frac{q}{2}, \frac{q}{2}+\frac{1}{2}, \frac{2 \mu R}{R_{0}}\right) \\
&+\frac{2^{q / 2-3}}{(q-2)(q-1) q \mu^{3}}\left(\frac{\mu R}{R_{0}}\right)^{-q / 2} \\
& \times e^{-\mu R / R_{0}} R_{0}^{3} R^{q-3}\left(\frac{\mu}{R_{0}}\right)^{q} \\
& \times\left(4 \mu^{2} \frac{R^{2}}{R_{0}^{2}}+\frac{2 q \mu R}{R_{0}}-q+q^{2}\right) \\
&\left.\times W M\left(\frac{q}{2}+1, \frac{q}{2}+\frac{1}{2}, \frac{2 \mu R}{R_{0}}\right)\right]
\end{aligned}
$$

$$
\begin{aligned}
\int \frac{1}{R}\left|\frac{d \psi_{1}}{d R}\right|^{2} d R=\frac{1}{8 R_{0}^{2} R^{4}}[ & -2 \alpha^{2} R^{2}+2 R_{0} \alpha R \sin \left(\frac{2 \alpha R}{R_{0}}\right) \\
& \left.-R_{0}^{2}+R_{0}^{2} \cos \left(\frac{2 \alpha R}{R_{0}}\right)\right],
\end{aligned}
$$

$$
\begin{aligned}
\int \frac{1}{R}\left|\frac{d \psi_{2}}{d R}\right|^{2} d R & \\
=\frac{\sin ^{2} \alpha}{120 R_{0}^{4} R^{5}}[ & 63 R_{0}^{4} \mu R e^{\left(-2 \mu\left(-R_{0}+R\right) / R_{0}\right)} \\
& -2 R_{0}^{3} \mu^{2} R^{2} e^{\left(-2 \mu\left(-R_{0}+R\right) / R_{0}\right)} \\
& +2 R_{0}^{2} \mu^{3} R^{3} e^{\left(-2 \mu\left(-R_{0}+R\right) / R_{0}\right)} \\
& -4 R_{0} \mu^{4} R^{4} e^{\left(-2 \mu\left(R_{0}+R\right) / R_{0}\right)}
\end{aligned}
$$

$$
\begin{aligned}
& +54 R_{0}^{5} e^{\left(-2 \mu\left(-R_{0}+R\right) / R_{0}\right)} \\
& \left.+8 \mu^{5} R^{5} e^{2 \mu} E i\left(1, \frac{2 \mu R}{R_{0}}\right)\right],
\end{aligned}
$$

$$
\begin{aligned}
\int\left|\frac{d^{2} \psi_{1}}{d R^{2}}\right|^{2} R d R= & \frac{\alpha^{4}}{2 R_{0}^{4}}\left[\log \left(\frac{\alpha R}{R_{0}}\right)-C i\left(\frac{2 \alpha R}{R_{0}}\right)\right] \\
& -\frac{\alpha^{2}}{R_{0}^{2} R^{2}} \cos \left(\frac{2 \alpha R}{R_{0}}\right)+\frac{\alpha}{R_{0} R^{3}} \sin \left(\frac{2 \alpha R}{R_{0}}\right) \\
& -\frac{1}{2 R^{4}}+\frac{1}{2 R^{4}} \cos \left(\frac{2 \alpha R}{R_{0}}\right),
\end{aligned}
$$$$
\int\left|\frac{d^{2} \psi_{2}}{d R^{2}}\right|^{2} R d R
$$$$
=\frac{\sin ^{2} \alpha}{32 R_{0}^{4} R^{5}} e^{2 \mu}\left[-90 R_{0}^{5} e^{\left(-2 \mu R / R_{0}\right)}\right.
$$$$
-135 R_{0}^{4} \mu R e^{\left(-2 \mu R / R_{0}\right)}
$$$$
-86 R_{0}^{3} \mu^{2} R^{2} e^{\left(-2 \mu R / R_{0}\right)}
$$$$
-10 R_{0}^{2} \mu^{3} R^{3} e^{\left(-2 \mu R / R_{0}\right)}
$$$$
-12 R_{0} \mu^{4} R^{4} e^{\left(-2 \mu R / R_{0}\right)}
$$$$
\left.+24 \mu^{5} R^{5} E i\left(1, \frac{2 \mu R}{R_{0}}\right)\right],
$$

$$
\begin{aligned}
\int\left|\frac{d^{3} \psi_{1}}{d R^{3}}\right|^{2} R d R & \\
= & \alpha^{6}\left[\frac{1}{2 R_{0}^{6}} \log \left(\frac{\alpha R}{R_{0}}\right)-\frac{1}{2 R_{0}^{6}} \operatorname{Ci}\left(\frac{2 \alpha R}{R_{0}}\right)\right] \\
& +\frac{\alpha^{5}}{2 R_{0}^{5} R} \sin \left(\frac{2 \alpha R}{R_{0}}\right) \\
& +\alpha^{4}\left[\frac{5}{4 R_{0}^{4} R^{2}} \cos \left(\frac{2 \alpha R}{R_{0}}\right)+\frac{3}{4 R_{0}^{4} R^{2}}\right]
\end{aligned}
$$

$$
\begin{aligned}
& -\frac{4 \alpha^{3}}{R_{0}^{3} R^{3}} \sin \left(\frac{2 \alpha R}{R_{0}}\right)-\frac{6 \alpha^{2}}{R_{0}^{2} R^{4}} \cos \left(\frac{2 \alpha R}{R_{0}}\right) \\
& +\frac{6 \alpha}{R_{0} R^{5}} \sin \left(\frac{2 \alpha R}{R_{0}}\right)+\frac{3}{R^{6}} \cos \left(\frac{2 \alpha R}{R_{0}}\right)-\frac{3}{R^{6}},
\end{aligned}
$$

$$
\begin{gathered}
\int\left|\frac{d^{3} \psi_{2}}{d R^{3}}\right|^{2} R d R R \\
=\frac{\sin ^{2} \alpha}{64 R_{0}^{6} R^{7}} e^{2 \mu}
\end{gathered}
$$




$$
\begin{aligned}
& \times\left[40 \mu^{7} R^{7} E i\left(1, \frac{2 \mu R}{R_{0}}\right)+1575 R_{0}^{7} e^{-\left(2 \mu R / R_{0}\right)}\right. \\
& +2625 R_{0}^{6} \mu R e^{-\left(2 \mu R / R_{0}\right)}+2082 R_{0}^{5} \mu^{2} R^{2} e^{-\left(2 \mu R / R_{0}\right)} \\
& +999 R_{0}^{4} \mu^{3} R^{3} e^{-\left(2 \mu R / R_{0}\right)}+246 R_{0}^{3} \mu^{4} R^{4} e^{-\left(2 \mu R / R_{0}\right)} \\
& \left.+42 R_{0}^{2} \mu^{5} R^{5} e^{-\left(2 \mu R / R_{0}\right)}-20 R_{0} \mu^{6} R^{6} e^{-\left(2 \mu R / R_{0}\right)}\right], \\
& \int \frac{1}{R}\left|\frac{d \psi_{1}}{d R}\right|\left|\frac{d^{3} \psi_{1}}{d R^{3}}\right| d R \\
& =\frac{1}{8 R_{0}^{4} R^{6}}\left[-4 R_{0}^{4}-3 \alpha^{2} R_{0}^{2} R^{2}+2 \alpha^{4} R^{4}\right. \\
& -2 \alpha^{3} R_{0} R^{3} \sin \left(\frac{2 \alpha R}{R_{0}}\right)+8 \alpha R_{0}^{3} R \sin \left(\frac{2 \alpha R}{R_{0}}\right) \\
& \left.-5 \alpha^{2} R_{0}^{2} R^{2} \cos \left(\frac{2 \alpha R}{R_{0}}\right)+4 R_{0}^{4} \cos \left(\frac{2 \alpha R}{R_{0}}\right)\right] \text {, } \\
& \int \frac{1}{R}\left|\frac{d \psi_{2}}{d R}\right|\left|\frac{d^{3} \psi_{2}}{d R^{3}}\right| d R \\
& =\frac{\sin ^{2} \alpha}{240 R_{0}^{6} R^{7} e^{\left(2 \mu R / R_{0}\right)}} e^{2 \mu} \\
& \times\left[975 R_{0}^{6} \mu R+474 R_{0}^{5} \mu^{2} R^{2}\right. \\
& +123 R_{0}^{4} \mu^{3} R^{3}-2 R_{0}^{3} \mu^{4} R^{4} \\
& +2 R_{0}^{2} \mu^{5} R^{5}-4 R_{0} \mu^{6} R^{6} \\
& +8 \mu^{7} R^{7} e^{\left(2 \mu R / R_{0}\right)} E i\left(1, \frac{2 \mu R}{R_{0}}\right) \\
& \left.+675 R_{0}^{7}\right] \\
& \left|\frac{d^{2} \psi}{d R^{2}}\right|=\frac{\sin \alpha}{4 R^{5}\left(R_{0} / R\right)^{3 / 2}} e^{\mu} e^{-\left(\mu R / R_{0}\right)} \\
& \times\left(15 R_{0}^{2}+12 R_{0} \mu R+4 \mu^{2} R^{2}\right),
\end{aligned}
$$

where $\psi_{1}$ and $\psi_{2}$ are given in (26). $\alpha, R_{0}$, and $\mu$ are the three variational parameters present in the fundamental modal solution.

$\operatorname{Ei}(z)$ is exponential integral given by [23] as follows:

$$
E i(1, z)=\int_{z}^{\infty} \frac{e^{-t}}{t} d t
$$

$\mathrm{Ci}(z)$ is the cosine integral function, defined by [23], as follows:

$$
C i(z)=\chi+\ln (z)+\int_{0}^{z} \frac{\cos t-1}{t} d t,
$$

where $\chi$ is Euler's constant 0.5772 .
$W M(k, m, z)$ are the Whittaker functions which are solutions to the Whittaker differential equation [23].

Hypergeom $(n, d, z)$ is the generalized hypergeometric function $F(n, d, z)$, where [23]

$$
F(n, d, z)=\sum_{k=0}^{\infty} \frac{C_{n, k}}{C_{d, k}} \cdot \frac{z^{k}}{k !}
$$

with,

$$
C_{v, k}=\prod_{j=1}^{v} \frac{\Gamma\left(v_{j}+k\right)}{\Gamma\left(v_{j}\right)},
$$

where $\Gamma(a)$ is the gamma function [23].

2.2. Basic Formulations. The refractive index profile for a weakly guiding fiber is given by

$$
\begin{array}{ll}
n_{f 1}^{2}=n_{2}^{2}+\left(n_{1}^{2}-n_{2}^{2}\right) f_{1}, & \text { for } 0 \leq R \leq S_{0}, \\
n_{f 2}^{2}=n_{2}^{2}+\left(n_{1}^{2}-n_{2}^{2}\right) f_{2}, & \text { for } S_{0} \leq R \leq 1, \\
n_{f 3}^{2}=n_{2}^{2}+\left(n_{1}^{2}-n_{2}^{2}\right) f_{3}, & \text { for } R>1,
\end{array}
$$

where the normalized profile functions for the trapezoidal and triangular index profiles $f_{i}(i=1,2,3)$ are given by

$$
\begin{aligned}
& f_{1}=1, \\
& f_{2}=\frac{1-R}{1-S_{0}} \\
& f_{3}=0 .
\end{aligned}
$$

Here, $S_{0}$ is the aspect ratio, $R$ is the normalized radius $(=r / a)$, $a$ is the core radius, $r$ is the actual radius of the optical fiber, and $n_{1}$ and $n_{2}$ are, respectively, the refractive indices of the core axis and cladding.

For the present study, the following approximations for the fundamental mode as the trial field have been proposed:

$$
\begin{aligned}
& \psi_{1}=\frac{\sin \left(\alpha R / R_{0}\right)}{R} \quad \text { for } R \leq R_{0}, \\
& \psi_{2}=\left(\frac{\sin (\alpha)}{R}\right) e^{\mu\left(1-\left(R / R_{0}\right)\right)} \sqrt{\frac{R_{0}}{R}} \text { for } R>R_{0},
\end{aligned}
$$

where $\alpha, R_{0}$, and $\mu$ are the three variational parameters present in the fundamental modal solution.

To employ variational technique, first the scalar variational expression for the propagation constant $\beta$ as given by 
(27) has been considered and is shown in equations through (28) to (30) as follows:

$$
\begin{gathered}
\beta^{2}=\frac{k^{2} \int_{0}^{\infty} n^{2}(R)|\psi(R)|^{2} R d R-\left(1 / a^{2}\right)\left\langle\psi^{\prime 2}\right\rangle}{\left\langle\psi^{2}\right\rangle} \\
\beta^{2}=\frac{1}{\left\langle\psi^{2}\right\rangle}\left[\int_{0}^{R_{0}} k^{2} n_{f 1}^{2}\left|\psi_{1}\right|^{2} R d R+\int_{R_{0}}^{S_{0}} k^{2} n_{f 1}^{2}\left|\psi_{2}\right|^{2} R d R\right. \\
+\int_{S_{0}}^{1} k^{2} n_{f 2}^{2}\left|\psi_{2}\right|^{2} R d R+\int_{1}^{\infty} k^{2} n_{f 3}^{2}\left|\psi_{2}\right|^{2} R d R \\
\left.-\frac{1}{a^{2}}\left\langle\psi^{\prime 2}\right\rangle\right]
\end{gathered}
$$

for $R_{0}<1$ and $R_{0}<S_{0}$,

$$
\begin{aligned}
\beta^{2}=\frac{1}{\left\langle\psi^{2}\right\rangle}[ & \int_{0}^{S_{0}} k^{2} n_{f 1}^{2}\left|\psi_{1}\right|^{2} R d R+\int_{S_{0}}^{R_{0}} k^{2} n_{f 2}^{2}\left|\psi_{1}\right|^{2} R d R \\
& +\int_{R_{0}}^{1} k^{2} n_{f 2}^{2}\left|\psi_{2}\right|^{2} R d R+\int_{1}^{\infty} k^{2} n_{f 3}^{2}\left|\psi_{2}\right|^{2} R d R \\
& \left.-\frac{1}{a^{2}}\left\langle\psi^{\prime 2}\right\rangle\right]
\end{aligned}
$$

for $R_{0}<1$ and $R_{0}>S_{0}$,

$$
\begin{aligned}
\beta^{2}=\frac{1}{\left\langle\psi^{2}\right\rangle} & {\left[\int_{0}^{S_{0}} k^{2} n_{f 1}^{2}\left|\psi_{1}\right|^{2} R d R\right.} \\
& +\int_{S_{0}}^{1} k^{2} n_{f 2}^{2}\left|\psi_{1}\right|^{2} R d R+\int_{1}^{R_{0}} k^{2} n_{f 3}^{2}\left|\psi_{1}\right|^{2} R d R \\
& \left.+\int_{R_{0}}^{\infty} k^{2} n_{f 3}^{2}\left|\psi_{2}\right|^{2} R d R-\frac{1}{a^{2}}\left\langle\psi^{\prime 2}\right\rangle\right]
\end{aligned}
$$

for $R_{0}>1$, where

$$
\begin{aligned}
\left\langle\psi^{2}\right\rangle & =\int_{0}^{R_{0}}\left|\psi_{1}\right|^{2} R d R+\int_{R_{0}}^{\infty}\left|\psi_{2}\right|^{2} R d R, \\
\left\langle\psi^{\prime 2}\right\rangle & =\int_{0}^{R_{0}}\left|\frac{d \psi_{1}}{d R}\right|^{2} R d R+\int_{R_{0}}^{\infty}\left|\frac{d \psi_{2}}{d R}\right|^{2} R d R .
\end{aligned}
$$

Now, the core parameter $U$ is given by

$$
U^{2}=a^{2}\left(k^{2} n_{1}^{2}-\beta^{2}\right)
$$

Now for a fixed value of normalized frequency, the core parameter $U$ is minimized with respect to the variational parameters $\alpha, R_{0}$, and $\mu$. Once the optimized values of these three parameters are obtained, the propagation constant and other design parameters can be obtained as explained in the next section.
2.3. Splice Loss. For small angular misalignment $(\theta)$ at the splice of two optical fibers, following Hosain et al. [11], the well-known overlap integral can be represented as

$$
C_{a}(p)=\int_{0}^{2 \pi} \int_{0}^{\infty} d \phi R d R|\psi(R)|^{2} \exp (i p R \cos \phi),
$$

where $p=a k n \theta, n$ being refractive index of the index matching fluid joining the fibers and $\theta$ being the angular misalignment.

The transmission coefficient $T_{a}(p)$ at the splice with angular mismatch can then be expressed as

$$
T_{a}(p)=\left|\frac{C_{a}(p)}{C_{a}(0)}\right|^{2} .
$$

Expanding the exponential term, (33) can be written as

$$
C_{a}(p)=2 \pi \sum_{n=0}^{n=\infty} \frac{\left(-p^{2} / 4\right)^{n}}{(n !)^{2}} \int_{0}^{\infty}|\psi(R)|^{2} R^{2 n+1} d R,
$$

and from (33), $C_{a}(0)$ is given by

$$
C_{a}(0)=2 \pi \int_{0}^{\infty} R d R|\psi(R)|^{2} .
$$

According to Hosain et al. [11], only the first four terms in (35a) are enough to obtain sufficient accuracy for misalignment up to $1^{0}$, which corresponds to $p \approx 0.8$ for an optical fiber with $n=1.5$ and $a=4 \mu \mathrm{m}$ working at a wavelength $\lambda=0.8 \mu \mathrm{m}$. Here, up to the fifth term of (35a) have been calculated and the required expressions are given in (9)-(10).

The transmission coefficient $T_{t}(\Delta)$ at the splice for a transverse offset $d$ is expressed as follows:

$$
T_{t}(\Delta)=\left|\frac{C_{t}(\Delta)}{C_{t}(0)}\right|^{2},
$$

where $\Delta=d / a$ is the normalized transverse offset, and in practice for $\Delta \leq 0.8$, one can approximately write [11]

$$
\frac{C_{t}(\Delta)}{C_{t}(0)}=1-\frac{B_{1}}{B_{0}}\left(\frac{\Delta}{2}\right)^{2}+\frac{B_{2}}{B_{0}}\left(\frac{\Delta}{2}\right)^{4}-\frac{B_{3}}{B_{0}}\left(\frac{\Delta}{2}\right)^{6},
$$

where

$$
\begin{gathered}
B_{0}=\left\langle\psi^{2}\right\rangle, \\
B_{1}=\left\langle\psi^{\prime 2}\right\rangle, \\
B_{2}=\frac{1}{4}\left(\int_{0}^{\infty}\left|\frac{d^{2} \psi}{d R^{2}}\right|^{2} R d R+\int_{0}^{\infty}\left|\frac{d \psi}{d R}\right|^{2} \frac{d R}{R}\right), \\
B_{3}=\frac{1}{36} \int_{0}^{\infty}\left|\frac{d^{3} \psi}{d R^{3}}\right|^{2} R d R-\frac{1}{12} \int_{0}^{\infty} \frac{d \psi}{d R} \frac{d^{3} \psi}{d R^{3}} \frac{d R}{R} \\
-\frac{1}{24}\left(\frac{d^{2} \psi}{d R^{2}}\right)_{R=0} \cdot
\end{gathered}
$$

Integrals given in (38)-(41) can be evaluated by using the expression of fundamental modal field given by (26). Hence, (34) and (36) can be evaluated with the help of (35a), (35b), and (37). 


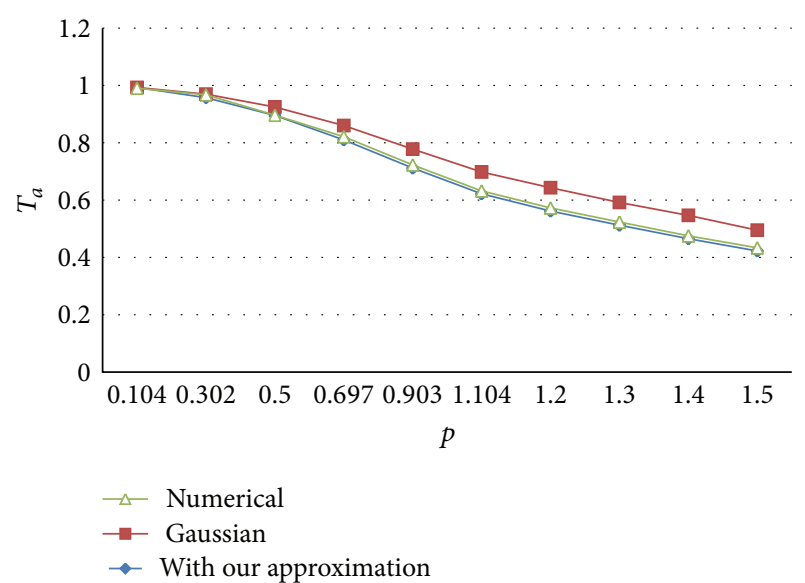

FIGURE 1: Variation of power transmission coefficients $T_{a}$ with the normalized angular offset $p$ for splicing of two identical single mode triangular index fibers with $V=2.7$ (exact numerical results [10, 13]; results by our approximation; results based on Gaussian approximation $[10,13])$.

2.4. Evaluation of Integrals. Evaluation of integrals to determine propagation constant and splice loss is presented in (1)(23). Substituting (1)-(6) into (28), (29), and (30), analytical expression of propagation constant $\beta$ can be obtained with the help of (26) and (27). The transmission coefficient $T_{a}(p)$ (34) at the splice with angular mismatch can be obtained by substituting (9)-(10) into (35a) and (35b). Using (31), (1), (2), (5), and (6), (38) can be obtained. Equations (40) and (41) can be evaluated using (11). Once (38)-(41) are evaluated, $C_{t}(\Delta) / C_{t}(0)$ (see (37)) can be calculated. Then, the analytical expression of transmission coefficient $T_{t}(\Delta)$ at the splice between two identical optical fibers having a transverse offset can be evaluated using (36).

\section{Results and Discussions}

Detailed comparison between the proposed formulation and available exact numerical results [10,13] has been carried out in terms of accuracy assessment. It has been justified by many authors [1-3] that two-parameter approximations are more accurate than single-parameter approximation. The proposed approximation of fundamental field involving three optimizing parameters incorporates more flexibility to modify the fundamental modal solution of optical fibers having different specifications. Optimized values of these parameters for different normalized frequencies are given in Tables 1 and 2 for a particular specification of optical fiber having trapezoidal and triangular index profiles, respectively. Values for other normalized frequencies having different specification of optical fiber can also be obtained by using Nelder-Mead method of nonlinear unconstrained minimization.

In order to verify the feasibility of the proposed approximation, the outcomes of the proposed study have been compared with the earlier reported numerical results $[10,13]$. In the present study, $S_{0}=0.25$ and $V=2.4$ are considered for trapezoidal index profile, which corresponds to a typical

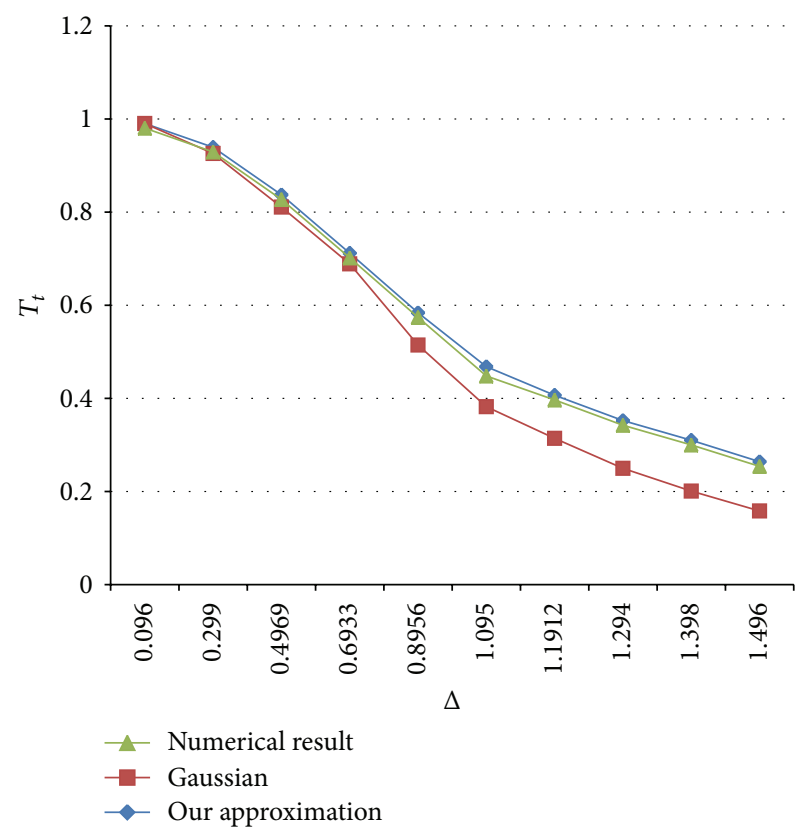

Figure 2: Variation of power transmission coefficients $T_{t}$ with the normalized transverse offset $\Delta$ for splicing of two identical single mode triangular index fibers with $V=2.7$ (exact numerical results $[10,13]$; results by our approximation; results based on Gaussian approximation $[10,13])$.

TABLE 1: Values of optimizing parameters with different normalized frequencies for trapezoidal index profile.

\begin{tabular}{lccc}
\hline$\alpha$ & $R_{0}$ & $\mu$ & $V$ \\
\hline 1.839506 & 1.487480 & 0.006634 & 1.6000 \\
1.854451 & 1.152568 & 0.040590 & 1.8000 \\
1.878781 & 1.006541 & 0.097612 & 2.0000 \\
1.908874 & 0.923490 & 0.171137 & 2.2000 \\
1.941083 & 0.867514 & 0.253638 & 2.4000 \\
1.954907 & 0.814689 & 0.289126 & 2.6000 \\
1.987346 & 0.781444 & 0.277328 & 2.8000 \\
1.967303 & 0.722072 & 0.256257 & 3.0000 \\
2.207612 & 0.797867 & 0.283156 & 3.2000 \\
1.930533 & 0.637208 & 0.226140 & 3.4000 \\
\hline
\end{tabular}

dispersion shifted silica fiber with $a=3.2 \mu \mathrm{m}, \delta=\left(n_{1}{ }^{2}-\right.$ $\left.n_{2}{ }^{2}\right) / 2 n_{1}{ }^{2}=0.008$, and zero dispersion wavelength at $1.55 \mu \mathrm{m}$ [12]. For triangular index profile, $V=2.7$ and $S_{0}=0$ have been chosen, taking $a=3.5 \mu \mathrm{m}, \delta=0.008$, and zero dispersion wavelength at $1.5 \mu \mathrm{m}$ [12].

For evaluation of splice loss, the applicability of the proposed formulations in case of power transmission coefficients $T_{a}(p)$ and $T_{t}(\Delta)$ at splices between two identical optical fibers has been considered. Gaussian approximation gives accurate result for the evaluation of transmission coefficient only in the region near the cutoff of single mode operation, but it leads to considerable error throughout the single mode region [11]. The variation of $T_{a}$ with $p$ and $T_{t}$ with $\Delta$, in case of splicing of two identical triangular index fibers, has been plotted in 


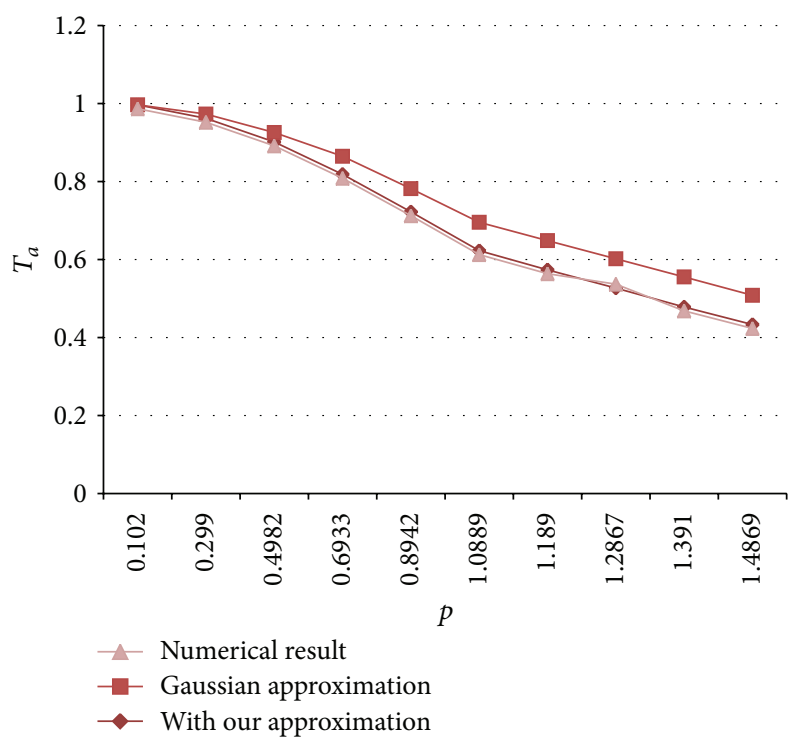

FIGURE 3: Variation of power transmission coefficients $T_{a}$ with the normalized angular offset $p$ for splicing of two identical single mode trapezoidal index fibers with $V=2.4$ (exact numerical results [10, 13]; results by our approximation; results based on Gaussian approximation $[10,13])$.

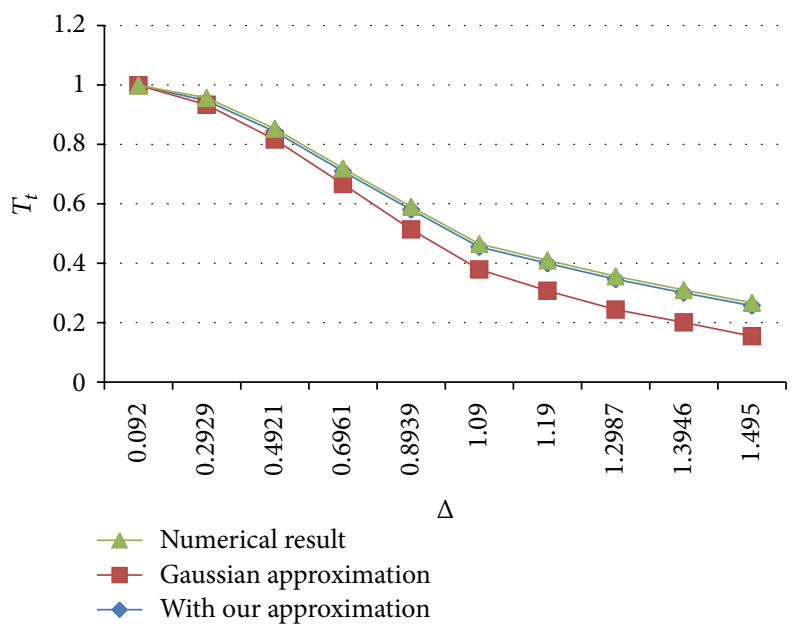

FIGURE 4: Variation of power transmission coefficients $T_{t}$ with the normalized transverse offset $\Delta$ for splicing of two identical single mode trapezoidal index fibers with $V=2.4$ (exact numerical results $[10,13]$; results by our approximation; results based on Gaussian approximation $[10,13])$.

Figures 1 and 2, respectively. Similarly, the variations of these power transmission coefficients for the case of splicing of two identical trapezoidal index fibers are illustrated in Figures 3 and 4 . For the practical range of $p$ and $\Delta$, the results obtained by the proposed approximation are identically matching with the exact available and numerical results $[10,13]$.
TABLE 2: Values of optimizing parameters with different normalized frequencies for triangular index profile.

\begin{tabular}{lccc}
\hline$\alpha$ & $R_{0}$ & $\mu$ & $V$ \\
\hline 1.836461 & 1.962401 & -0.000309 & 1.7000 \\
1.843169 & 1.323589 & 0.014877 & 1.9000 \\
1.858852 & 1.102224 & 0.050720 & 2.1000 \\
1.881018 & 0.986778 & 0.103042 & 2.3000 \\
1.907224 & 0.914502 & 0.167036 & 2.5000 \\
1.934891 & 0.862840 & 0.237307 & 2.7000 \\
1.954757 & 0.817735 & 0.290207 & 2.9000 \\
1.947828 & 0.764272 & 0.271233 & 3.1000 \\
1.941593 & 0.718887 & 0.255127 & 3.3000 \\
2.150373 & 0.784010 & 0.278234 & 3.5000 \\
\hline
\end{tabular}

\section{Conclusions}

An accurate three-parameter approximation of fundamental modal field solution of an optical fiber has been presented, which can effectively be used to estimate the power transmission coefficients in case of splicing of two identical single mode graded-index fibers in presence of both transverse and angular misalignments. Taking trapezoidal and triangular index fibers as examples, it has been shown that the results obtained with our function are excellently matching with the exact available and numerical results [10, 13]. Besides providing values of optimizing parameters involved in the approximate field obtained by Nelder-Mead method of nonlinear unconstrained minimization, all related simplified analytical expressions have also been presented, which can be used directly by optical fiber designer while predicting splice losses of an optical fiber, having triangular and trapezoidal index profiles for a wide range of normalized frequencies. The salient features of the proposed solution are easy computation on an ordinary personal computer and a robust algorithm for nonlinear unconstrained optimization being applied in an optical fiber having triangular and trapezoidal index profiles.

\section{References}

[1] R. Tewari, S. I. Hosain, and K. Thyagarajan, "Scalar variational analysis of single mode fibers with Gaussian and smoothedout profiles," Optics Communications, vol. 48, no. 3, pp. 176-180, 1983.

[2] A. Sharma, S. I. Hosain, and A. K. Ghatak, "The fundamental mode of graded-index fibres: simple and accurate variational methods," Optical and Quantum Electronics, vol. 14, no. 1, pp. 7-15, 1982.

[3] A. Sharma and A. K. Ghatak, "A variational analysis of single mode graded-index fibers," Optics Communications, vol. 36, no. 1, pp. 22-24, 1981.

[4] A. Ankiewicz and G.-D. Peng, "Generalized Gaussian approximation for single-mode fibers," Journal of Lightwave Technology, vol. 10, no. 1, pp. 22-27, 1992.

[5] S. Chieh, W.-H. Tsai, and M.-S. Wu, "Extended Gaussian approximation for single-mode graded-index fibers," Journal of Lightwave Technology, vol. 12, no. 3, pp. 392-395, 1994. 
[6] G. De Angelis, G. Panariello, and A. Scaglione, "Variational method to approximate the field of weakly guiding optical fibers by Laguerre-Gauss/Bessel expansion," Journal of Lightwave Technology, vol. 17, no. 12, pp. 2665-2674, 1999.

[7] F. Chiadini, G. Panariello, and A. Scaglione, "Variational analysis of matched-clad optical fibers," Journal of Lightwave Technology, vol. 21, no. 1, pp. 96-105, 2003.

[8] Q. Cao and S. Chi, "Approximate analytical description for fundamental-mode fields of graded-index fibers: beyond the Gaussian approximation," Journal of Lightwave Technology, vol. 19, no. 1, pp. 54-59, 2001.

[9] A. K. Ghatak, R. Srivastava, I. F. Faria, K. Thyagarajan, and R. Tiwari, "Accurate method for characterising single-mode fibres: theory and experiment," Electronics Letters, vol. 19, no. 3, pp. 9799, 1983.

[10] J. P. Meunier and S. I. Hosain, "An efficient model for splice loss evaluation in single-mode graded-index fibers," Journal of Lightwave Technology, vol. 9, no. 11, pp. 1457-1463, 1991.

[11] S. I. Hosain, A. Sharma, and A. K. Ghatak, "Splice-loss evaluation for single-mode graded-index fibers," Applied Optics, vol. 21, no. 15, pp. 2716-2720, 1982.

[12] U. C. Paek, "Dispersionless single-mode fibers with trapezoidalindex profiles in the wavelength region near $1.5 \mu \mathrm{m}$," Applied Optics, vol. 22, no. 15, pp. 2363-2369, 1983.

[13] K. Kawano and T. Kitoh, Introduction to Optical Waveguide Analysis, John Willy and Sons, New York, NY, USA, 2001.

[14] J. C. Lagarias, J. A. Reeds, M. H. Wright, and P. E. Wright, "Convergence properties of the Nelder-Mead simplex method in low dimensions," SIAM Journal on Optimization, vol. 9, no. 1, pp. 112-147, 1998.

[15] J. M. Parkinson and D. Hutchinson, "An investigation into the efficiency of variants on the simplex method," in Numerical Methods for Nonlinear Optimization, F. A. Lootsma, Ed., pp. 115135, Academic Press, New York, NY, USA, 1972.

[16] M. H. Wright, "Direct search methods: once scorned, now respectable," in Proceedings of the Dundee Biennial Conference in Numerical Analysis, D. F. Griffiths and G. A. Watson, Eds., pp. 191-208, Addison Wesley; Longman, Harlow, UK, 1996.

[17] J. A. Nelder and R. Mead, "A simplex method for function minimization," Computer Journal, vol. 7, pp. 308-313, 1965.

[18] T. H. Rowan, Functional stability analysis of numerical algorithms [Ph.D. thesis], University of Texas, Austin, Tex, USA, 1990.

[19] S. Singer and S. Singer, "Complexity analysis of Nelder-Mead search iterations," in Proceedings of the 1st Conference on Applied Mathematics and Computation, Dubrovnik, Croatia, 1999, M. Rogina, V. Hari, N. Limić, and Z. Tutek, Eds., pp. 185-196, PMFMatematički odjel, Zagreb, 2001.

[20] S. Singer and S. Singer, "Efficient implementation of the NelderMead search algorithm," Applied Numerical Analysis and Computational Mathematics, vol. 1, no. 3, pp. 524-534, 2004.

[21] R. Roychoudhury and A. Roychoudhury, "Accurate semi analytical model of an optical fiber having Kerr nonlinearity using a robust nonlinear unconstrained optimization method," Optics Communications, vol. 284, no. 4, pp. 1038-1044, 2011.

[22] I. S. Gradshteyn and I. M. Ryzhik, Table of Integrals, Series and Products, Academic Press, New York, NY, USA, 1980.

[23] M. Abramowitz and I. A. Stegun, Handbook of Mathematical Functions, Dover, New York, NY, USA, 1981. 

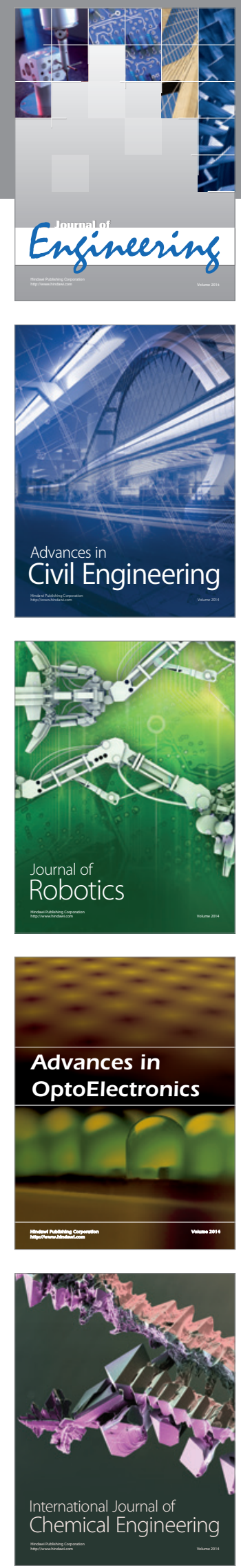

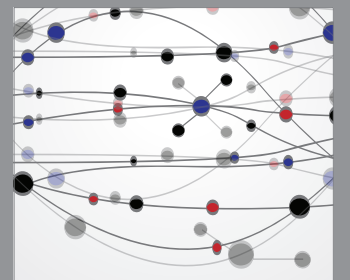

The Scientific World Journal
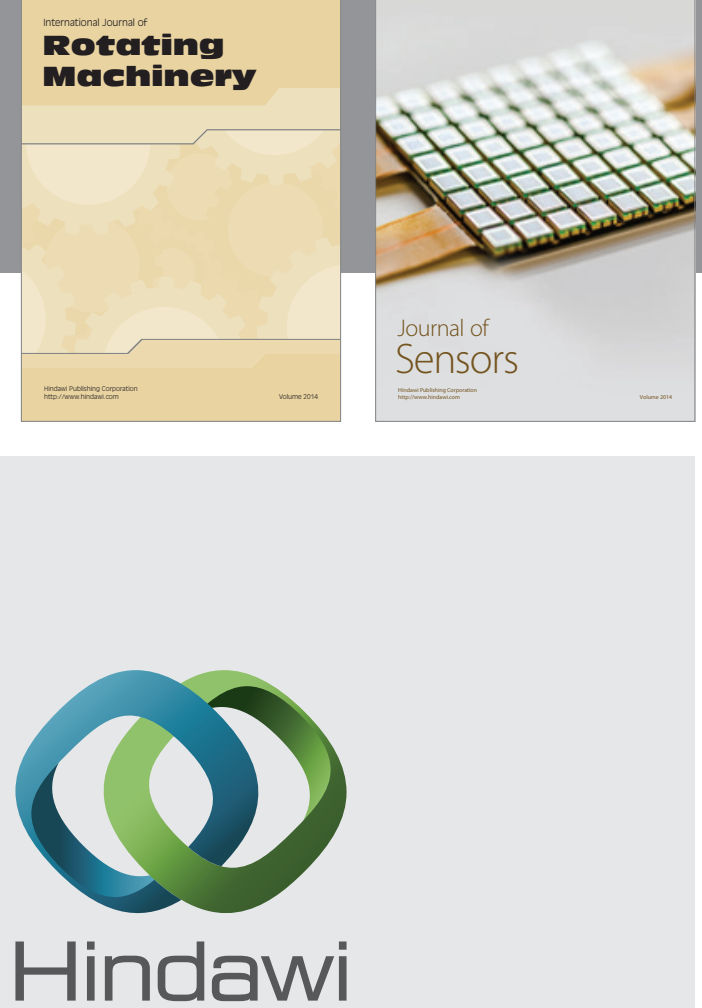

Submit your manuscripts at http://www.hindawi.com
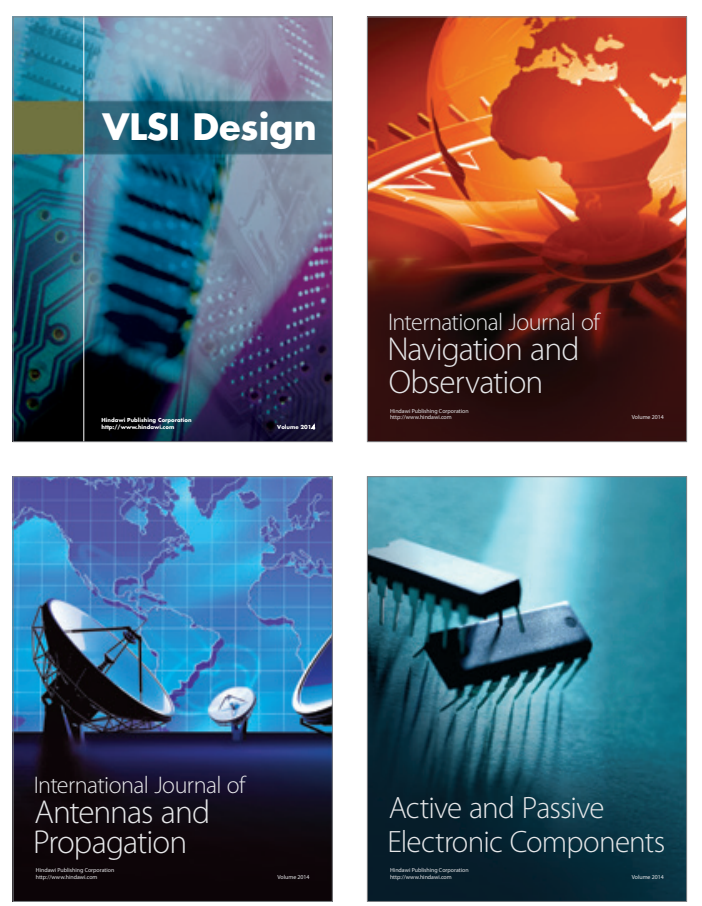
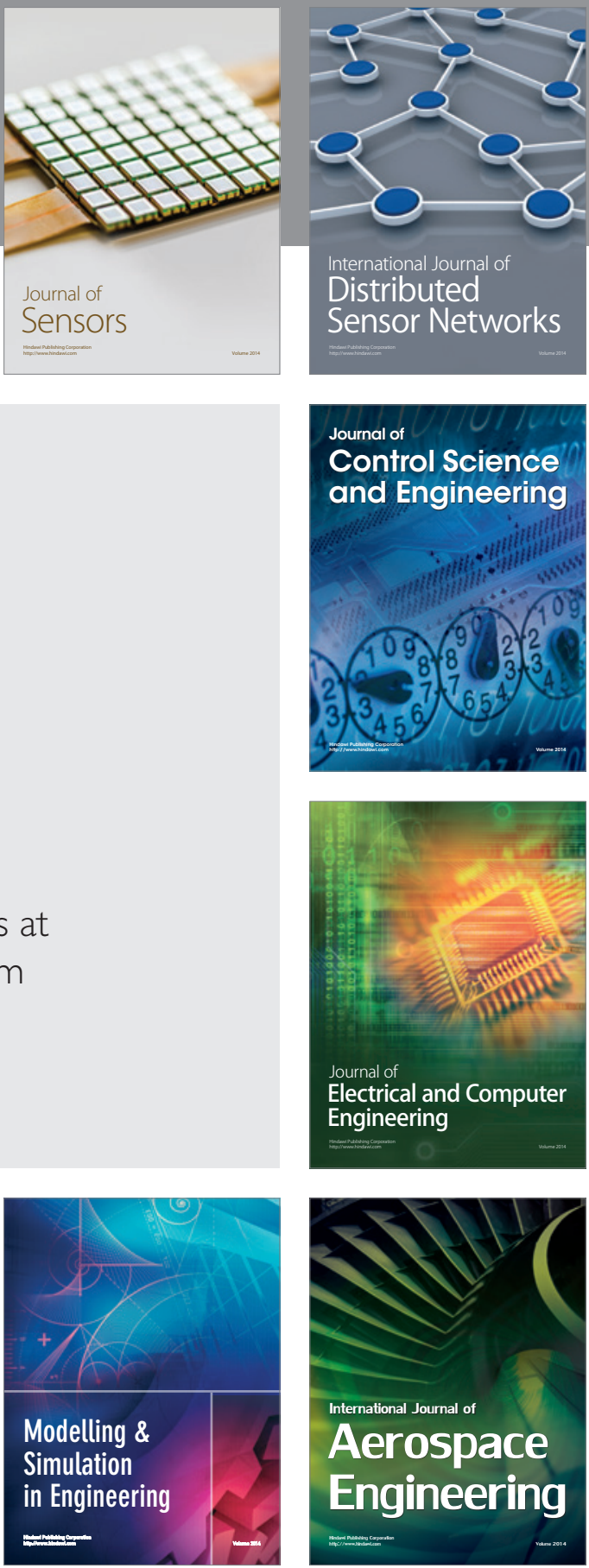

Journal of

Control Science

and Engineering
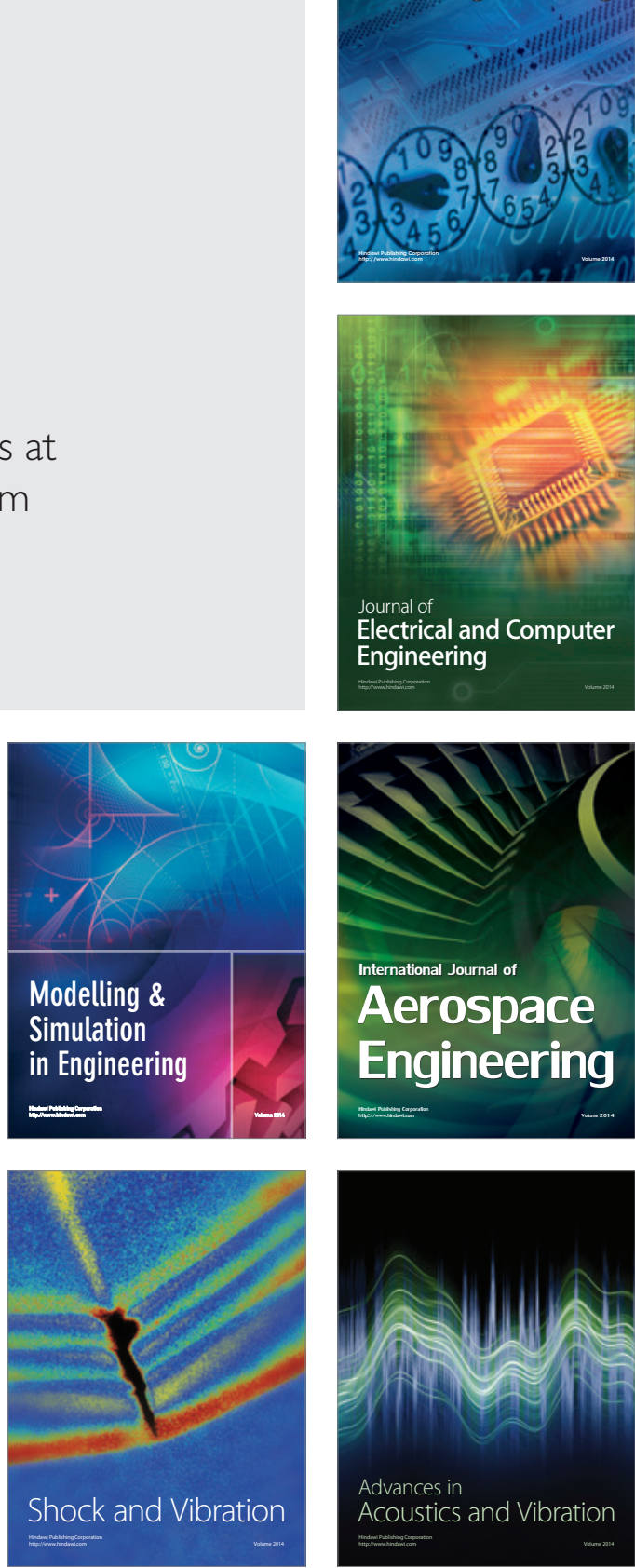